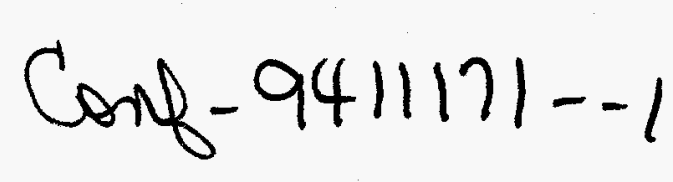

SLAC-PUB-6509

August 1994

(T/Noyes)

\title{
Bit-String Physics: a Novel "Theory of Everything"*
}

\author{
H. Pierre Noyes \\ Stanford Linear Accelerator Center, Stanford University, Stanford, CA 94309
}

\begin{abstract}
We encode the quantum numbers of the standard model of quarks and leptons using constructed bitstrings of length 256. These label a growing universe of bit-strings of growing length that eventually construct a finite and discrete space-time with reasonable cosmological properties. Coupling constants and mass ratios, computed from closure under XOR and a statistical hypothesis, using only $\hbar, c$ and $m_{p}$ to fix our units of mass, length and time in terms of standard (meterkilogram-second) metrology, agree with the first four to seven significant figures of accepted experimental results. Finite and discrete conservation laws and commutation relations ingure the essential characteristics of relativistic quantum mechanics, including particle-antiparticle pair creation. The correspondence limit in (free space) Maxwell electromagnetism and Einstein gravitation is consistent with the FeynmanDyson-Tanimura "proof."
\end{abstract}

Although currently accepted relativistic quantum mechanical theories incorporate many discrete phenomena, they are embedded in an underlying spacetime continuum in a way which guarantees the creation of infinities. Despite many phenomenological successes, they have as yet failed to achieve a consensus theory of "quantum gravity". We believe that these two difficulties are connected, and that both can be circumvented by basing fundamental physical theory directly on the computer tools of bit-strings and information theory based on bit-strings. This has the further advantage that we can base our model for space and time on finite intervals between events (eg., counter firings) measured to finite (and fixed in any particular context) accuracy. This operational methodology then allows us to avoid such metaphysical questions as whether the "real world" is discrete or continuous [1], or whether the "act of observation" does or does not require "consciousness" [2].

By a "theory of everything" (ToE), we mean a systematic representation of the numerical results obtained in high energy particle physics experiments and by observational cosmology. The representation we use employs a growing but always finite assemblage of bit-strings of finite length constructed by a simple algorithm called program universe explained below.

\footnotetext{
*Work supported by Department of Energy contract

DE-ACO3-76SF00515.
}

More conventional ToE's are based on the mathematical continuum and the structures of second quantized relativistic field theories (QFT). They ignore the flaws of QFT (infinite answers to physically sensible questions, unobservable "gauge potentials", and no well defined correspondence limit in either classical relativistic field theory, non-relativistic quantum mechanics or nuclear physics). The most ambitious of these theories assume that non-Abelian gauge theories in the form of "string theory" succeed in explaining "quantum gravity". Comparison with practical metrology is made by identifying $\hbar, c$ and $G_{N e w t o n}$ in their theoretical structures. It is then an act of faith that everything else is calculable. Less ambitious ToE's (eg., GUT's = grand unified theories) fix the third parameter as a universal coupling constant at an energy of about a thousandth of the Planck mass-energy and then "run" it down in three different ways to energies a factor of $10^{15}$ smaller where these three distinct values are identified as the measurable fine structure constant $\left(\alpha=e^{2} / \hbar c\right)$, weak interaction constant $\left(G_{F e r m i}\right)$ and strong coupling constant $\alpha_{s}$; because the strong (QCD) coupling "constant" is supposed to diverge at zero energy, models must include its energy dependence over a finite energy range. In practice, such theories contain a fairly large number of phenomenological parameters.

In contrast, we employ a structure in which we need only identify $\hbar, c$ and $m_{p}$ (the proton mass) in order to make contact with standard MLT metrology, using the kilogram, meter, and second as arbitrary but fixed dimensional units. $\alpha, G_{\text {Fermi }}, G_{\text {Newton }}$ and a number of other well measured parameters can be computed and the quality of the fit to experiment evaluated in a less problematic way. While these comparisons are very encouraging, with accuracies ranging from four to seven significant figures, they are not perfect. So far as we can see the discrepancies could arise from the concatenation of effects we know we have so far not included in the calculations, but we are prepared to encounter "failure" as we extend the calculations. However, the quality of the results achieved to date lead us to expect that such "failure" would point to where to look for "new physics" in our sense. Since we leave no place for "adjustable parameters", such a crisis should be more clear cut for us than in a conventional ToE. We do not believe that it is possible

Presented at the Workshop on Physics and Computation (PhysComp'94):

This Decade and Beyond, Dallas, TX, November 17-20, 1994 
to make a "final theory", and might even welcome a failure serious enough to allow us to abandon this whole approach and turn to more conventional activities.

We start from a universe of bit-strings of the same length which grow in length by a random bit, randomly chosen for each string whenever XOR between two strings gives the null string; else the resulting non-null string is adjoined to the universe. Then recurse. Because of closure under XOR [1], and a mapping we present below of the quantum numbers of the 3generation standard model of quarks and leptons onto the first 16 bits in these strings, we can model discrete quantum number conservation (lepton number, baryon number,charge, weak isospin, and color) using a bitstring equivalent of 4-leg Feynman diagrams. Quarks and color are necessarily confined. All known elementary fermions and bosons are generated, and no unknown particles are predicted. The scheme implies reasonably accurate coupling constants and mass ratios, calculated assuming equal prior probabilities in the absence of further information. The combinatorics and the standard statistical method of assigning equal weights to each possibility provide an alternative interpretation of results previously obtained from the combinatorial hieranchy, including the closure of these bit-string labels at length 256 , and the prediction of the Newtonian gravitational constant. Baryon and lepton number conservation then gravitationally stabilizes the lightest charged (free) baryon (the proton) and lepton (the electron) as rotating black holes of spin $1 / 2$ and unit charge.

The growing portion of the bit-strings beyond the quantum number conserving labels can be interpreted as describing an expanding 3-space universe with a universal (cosmological) time parameter. Within this universe pairwise collisions produce products conserving relativistic 3-momentum (and, when on mass shell, energy) in terms of quantized Mandelstam parameters and masses. The baryon and lepton number, ratio of baryons to photons, fireball time, and ratio of dark to baryonic matter predicted by this cosmological model are in rough accord with observation. The model contains the free space Maxwell equations for electromagnetism and the free space Einstein equations for gravitation as appropriate macroscopic approximations for computing the motion of a single test particle.

\section{Bit-strings and program universe}

Specify a bit-string a(S) by its $S$ ordered elements $a_{s} \in 0,1$; and $s \in 1,2,3, \ldots S$. If we interpret the symbols " 0 " and " 1 " in the strings as integers, we can calculate the norm, or Hamming measure, $a(S)$ by the formula $a \equiv \Sigma_{s=1}^{S} a_{s}$. We also call this positive integer both $|\mathbf{a}(S)|$ and $\langle\mathbf{a}(S) \mid \mathbf{a}(S)\rangle$. Here we introduce the Dirac bra (| notation for one dimensional row matrices, ket |\rangle for one dimensional column matrices, and braket $\langle\mid\rangle$ for the matrix inner product. Hence, $\langle\mathbf{a}(S) \mid \mathbf{b}(S)\rangle=$ $\Sigma_{s=1}^{S} a_{s} b_{s}$. Because we interpret the symbols " 0 " and " 1 " as integers rather than bits, we can define the operator XOR, symbolized by $\oplus$, which combines two strings to form a third by the elements of the resulting string: $(\mathbf{a} \oplus \mathbf{b})_{s} \equiv\left(a_{s}-b_{s}\right)^{2}$. This is isomorphic to the usual meaning of XOR, addition mod 2 or boolean symmetric difference in the sense that the element is 1 if $a_{s}$ and $b_{s}$ differ, and 0 if they are the same. In our restricted (bit-string) environment, the Dirac notation then allows us to write $|\mathbf{a} \oplus \mathbf{b}|+2\langle\mathbf{a} \mid \mathbf{b}\rangle=a+b$.

One consequence of this connection between bitstring states and bit-strings is that if $|\mathbf{a} \oplus \mathbf{b}|=$ $a+b$, then the states are orthogonal in the sense that $\langle\mathbf{a} \mid \mathbf{b}\rangle=0$. Hence given $n$ finite integers $n_{i}$ with the constraint $\sum_{i=1}^{n} n_{i}<S$, we can always construct an orthogonal state basis from $n$ bitstrings $\mathbf{n}_{i}(S)$ with a standard representation $\mathbf{n}_{i}(S) \equiv$ $\mathbf{0}\left(\Sigma_{j=1}^{n_{i-1}} n_{j}\right)\left\|\mathbf{I}\left(n_{i}\right)\right\| \mathbf{0}\left(S-\Sigma_{j=n_{i+1}}^{n} n_{j}\right)$. Here we have introduced the null string $0(W)$ with elements $0_{w}=0$, the anti-null string $\mathbf{I}(W)$ with elements $I_{w}=1$, and bit-string concatenation "\|" defined by $e_{k}^{a \| b} \equiv a_{k}, k \in$ $1,2, \ldots, S_{a} ; e_{k}^{a \| b} \equiv b_{j}, j \in 1,2, \ldots, S_{b}, k=S_{a}+j$. Given such a basis, we have $n$ strings which are independent under XOR and which can be combined by XOR to produce $2^{n}-1$ distinct strings simply by taking them $1,2, \ldots$ up to $n$ at a time.

To generate a growing universe of bit-strings which at each step contains $P(S)$ strings of length $S$, we use an algorithm known as program universe which was developed in collaboration with M.J.Manthey . $[2,3]$

Pick any two strings $\mathbf{P}_{i}(S), \mathbf{P}_{j}(S), i, j \in 1,2, \ldots, P$ and compare $\mathbf{P}_{i j}=\mathbf{P}_{\mathbf{i}} \oplus \mathbf{P}_{\mathbf{j}}$ with $\mathbf{0}(S)$.

If $\mathbf{P}_{i j} \neq \mathbf{0}$, adjoin $\mathbf{P}_{P+1}:=\mathbf{P}_{i j}$ to the universe, set $P:=P+1$ and recurse.

Else, for each $i \in 1,2, \ldots, P$ pick an arbitrary bit $\mathbf{a}_{i} \in 0,1$, replace $\mathbf{P}_{i}(S+1):=\mathbf{P}_{i}(S) \| \mathbf{a}_{i}$, set $S:=S+1$ and recurse.

Note that, because the arbitrary bits are concatenated only at one growing end of the strings, once the string length $S$ passes any fixed length $L$ the $P(L)$ strings present at that time can contain at most $L$ strings which are independent under XOR. Further, since this portion changes only by XOR once $S>L$, it can end up containing contain at most $2^{L}-1$ types of distinct, non-null strings. Consequently, at any later stage in the evolution of the universe we can always separate any string into two portions, a label string $\mathrm{N}_{i}(L)$ and a content string $\mathrm{C}_{i}(S-L)$ and write $P_{i}(S)=$ $\mathbf{N}_{i}(L) \| \mathbf{C}_{i}(S-L)$. 


\section{DISCLAIMER}

This report was prepared as an account of work sponsored by an agency of the United States Government. Neither the United States Government nor any agency thereof, nor any of their employees, makes any warranty, express or implied, or assumes any legal liability or responsibility for the accuracy, completeness, or usefulness of any information, apparatus, product, or process disclosed, or represents that its use would not infringe privately owned rights. Reference herein to any specific commercial product, process, or service by trade name, trademark, manufacturer, or otherwise does not necessarily constitute or imply its endorsement, recommendation, or favoring by the United States Government or any agency thereof. The views and opinions of authors expressed herein do not necessarily state or reflect those of the United States Government or any agency thereof. 


\section{DISCLAIMER}

Portions of this document may be illegible in electronic image products. Images are produced from the best available original document. 


\section{Bit-string standard model labels}

To model the quantum numbers of the standard model of quarks and leptons with label strings of length 16, we break the label into four segments: $\mathbf{L}_{S M}=$ $\mathbf{L}_{\nu}(2)\left\|\mathbf{L}_{\ell}(4)\right\| \mathbf{L}_{q}(8) \| \mathbf{L}_{g}(2)$. Here $g$ stands for the three generations of neutrinos, charged leptons and quarks with the standard designations $\left(\nu_{e}, e ; u, d\right),\left(\nu_{\mu}, \mu ; c, s\right)$, $\left(\nu_{\tau}, \tau ; t, b\right)$. The generation label $\mathbf{L}_{g}(2)$ has only three non-null possibilities $(10),(01),(11)$; the most convenient choice will depend on an investigation of the Kobiyashi-Maskawa mixing angles which has yet to be carried out. So far as we know, baryon number, lepton number, charge, and the $z$-component of weak isospin are conserved (and constrained to three independent choices by the generalized GellMann-Nishijima rule). We use the weak interaction to define the basis strings we take to be orthogonal and choose $L_{\nu}: \nu_{L}=$ $(10) ; \nu_{R}=(01)$. Then take $L_{\ell}: \ell_{L}^{-}=(1000) ; \ell_{L}^{+}=$ $(0100) ; \ell_{R}^{-}=(0010) ; \ell_{R}^{+}=(0001)$. For the quarks use $\mathbf{L}_{q_{u}}=\mathbf{q}(4)\left\|\mathbf{q}(4) ; \mathbf{L}_{q_{d}}=\mathbf{q}(4)\right\| \mathbf{0}(4)$ with $\mathbf{q}_{L, R}^{ \pm}$following the same pattern as the charged leptons.

The basic weak interaction vertices are then given by $\nu_{L} \oplus \mathbf{f}_{L}^{ \pm}=\mathbf{W}_{L L}^{ \pm} ; \nu_{L} \oplus \nu_{R}=\mathbf{Z}_{0}=\mathbf{f}_{L}^{\mp} \oplus \mathbf{f}_{L}^{ \pm}$where $f_{L}^{ \pm}$stands for any left-handed, charged fermion (i.e charged lepton or quark). Similarly, the electromagnetic vertices are given by $\mathbf{f}_{L}^{\mp} \oplus \mathbf{f}_{L}^{ \pm}=\gamma_{L L} ; \mathbf{f}_{L}^{\mp} \oplus \mathbf{f}_{R}^{ \pm}=$ $\gamma_{c}$. The $\gamma$ is massless, and the $Z_{0}$ is massive (and unstable), so we need to develop the content labels in terms of space-time parameters to distinguish $\gamma$ from $Z_{0}$. Note that the $Z_{0}$ and the $W$ couple only to left-handed fermions. The masslessness of the $\gamma$ 's keeps the transverse photons from coupling directly to the coulomb interaction $\gamma_{c}$, which is represented within the electromagnetic sector by (1111). Details, and proof of the conservation laws will be presented elsewhere.

This scheme gives us 137 distinct charged particles which can couple via the coulomb interaction in one, and only one, way. Thus, in the absence of further information, the probability of the coulomb scattering process $f^{ \pm}+f^{\mp} \rightarrow \gamma_{c} \rightarrow f^{ \pm}+f^{\mp}$ is $1 / 137$ and can be identified as the value of $e^{2} / \hbar c$ in the quantum number part of the corresponding Feynman diagram. That this leads to a correct description of the energy levels of the (relativistic) Bohr atom, and that the Sommerfeld fine structure is the appropriate $1 / 137$ correction to this result has been argued elsewhere. [4] Our present analysis needs rethinking before it can be claimed that it leads to McGoveran's correction to the calculated value of $e^{2} / \hbar c$ given in the table. Similarly, since (in the absence of further information) the four-fermion (weak) interaction can start from any pair of 16 strings and lead to any other pair, the a priori coupling probability is $1 /(256)^{2}$. Examination of how the
Fermi constant is actually evaluated in neutron decay then allows us to predict that $G_{F}=m_{p}^{-2} / \sqrt{2}(256)^{2}$. The correction given in the table is again due to McGoveran; as just noted, he invokes a different theory.

The quark-gluon (QCD) interaction requires a more careful discussion, which can only be sketched here. Thanks to McGoveran's Theorem [5] that in any finite and discrete theory with a universal ordering operator, there can be no more than three homogeneous and isotropic macroscopic ("spacial") dimensions, these are exhausted in labeling particles by the three absolutely conserved quantum numbers allowed in current particle physics. There are four candidates: lepton number, baryon number, charge, and either the "z-component of weak isospin" or "weak hypercharge". The extended GellMann-Nishijima relation leaves only three of these to specify independently and measure at macroscopic distances. Any other conserved, discrete quantum number must "compactify" or be "confined". The two flavors of quarks with the $L, R$ and quark-antiquark dichotomies give us 8 degrees of freedom in our basis, but the special role played by the anti-null string reduces these to seven which generate $2^{7}-1$ distinct non-null strings. These can be used to represent the $2^{4}$ possible quark and anti-quark states coupled to the color octet (red, anti-red, yellow, anti-yellow, blue, anti-blue, black, colorless $=2^{3}$ ) of gluons, or the requisite $2^{4} \times 2^{3}$ distinct quark and gluon states needed to describe strong (QCD) interactions. Color, gluons, and quarks are confined, thanks to McGoveran's theorem. The label scheme above, appropriately interpreted, provides the $2 / 3$ and $1 / 3$ charge and baryon number values for the up-type and down-type quarks, respectively.

To obtain the pion masses (given the electron mass-see below), we use Dyson's contention [6] that QED does not allow more than 137 charged particle pairs of mass $m$ to be specified within a volume whose radial dimension is $\hbar / 2 m c$. This allows us to model the neutral pion as $\hbar c / e^{2}=137$ electron-positron pairs within this distance, and the two charged pions as an electron (positron) and anti-neutrino (neutrino) included in this ephemeral structure [7]. The combinatorial corrections due to McGoveran and given in the table bring the calculated values reasonably close to experiment. We emphasize again that the legitimacy of using his corrections in our context is still controversial.

\section{Background}

We have used bit-string physics to provide a theory in which the approximation $\hbar c / e^{2}=2^{2}-1+2^{3}-$ $1+2^{7}-1=137$ makes sense in terms of a computer algorithm and information theory. Historically, the research program which originally led to this way of calculating a good approximation to the fine 
Table I. Coupling constants and mass ratios predicted by the finite and discrete unification of quantum mechanics and relativity. Empirical Input: $c, \hbar$ and $m_{p}$ as understood in the "Review of Particle Properties", Particle Data Group, Physics Letters, B 239, 12 April 1990.

\section{COUPLING CONSTANTS}

Coupling Constant

Calculated

$$
\begin{gathered}
G^{-1} \frac{\hbar c}{m_{p}^{2}} \\
G_{F} m_{p}^{2} / \hbar c \\
\sin ^{2} \theta_{W e a k} \\
\alpha^{-1}\left(m_{e}\right) \\
G_{\pi N \bar{N}}^{2}
\end{gathered}
$$$$
\left[2^{127}+136\right] \times\left[1-\frac{1}{3 \cdot 7 \cdot 10}\right]=1.69331 \ldots \times 10^{38}
$$$$
\left[256^{2} \sqrt{2}\right]^{-1} \times\left[1-\frac{1}{3 \cdot 7}\right]=1.02758 \ldots \times 10^{-5}
$$$$
0.25\left[1-\frac{1}{3 \cdot 7}\right]^{2}=0.2267 \ldots
$$$$
137 \times\left[1-\frac{1}{30 \times 127}\right]^{-1}=137.0359674 \ldots
$$$$
\left[\left(\frac{2 M_{N}}{m_{\pi}}\right)^{2}-1\right]^{\frac{1}{2}}=[195]^{\frac{1}{2}}=13.96 .
$$

\section{MASS RATIOS}

Mass ratio

$$
\text { Calculated }
$$

$$
m_{p} / m_{e}
$$

$$
m_{\pi}^{ \pm} / m_{e}
$$$$
m_{\pi^{0}} / m_{e}
$$$$
m_{\mu} / m_{e}
$$

$$
\begin{gathered}
\frac{137 \pi}{\frac{3}{14}\left(1+\frac{2}{7}+\frac{4}{49}\right) \frac{4}{5}}=1836.151497 \ldots \\
275\left[1-\frac{2}{2 \cdot 3 \cdot 7 \cdot 7}\right]=273.1292 \ldots \\
274\left[1-\frac{3}{2 \cdot 3 \cdot 7 \cdot 2}\right]=264.2143 \ldots \\
3 \cdot 7 \cdot 10\left[1-\frac{3}{3 \cdot 7 \cdot 10}\right]=207
\end{gathered}
$$

$\left[1.69358(21) \times 10^{38}\right]$

$\left[1.02682(2) \times 10^{-5}\right]$

$[0.2259(46)]$

[137.0359 895(61)]

$[13,3(3),>13.9 ?]$

\section{COSMOLOGICAL PARAMETERS}

Parameter

$$
\begin{gathered}
N_{B} / N_{\gamma} \\
M_{d a r k} / M_{v i s} \\
N_{B}-N_{\bar{B}} \\
\rho_{/} \rho_{c r i t}
\end{gathered}
$$

Calculated

$$
\begin{gathered}
\frac{1}{256^{4}}=2.328 \ldots \times 10^{-10} \\
\approx 12.7 \\
\left(2^{127}+136\right)^{2}=2.89 \ldots \times 10^{78} \\
\approx \frac{4 \times 10^{79} m_{p}}{M_{\text {crit }}}
\end{gathered}
$$

Observed

[1836.15 2701(37)]

[273.12 67(4)]

[264.1 373(6)]

[206.768 26(13)] 
structure constant was started by Ted Bastin and Clive Kilmister in the 1950s using a somewhat different set of computational ideas. They were joined by John Amson, Gordon Pask, and Fredrick Parker-Rhodes. In 1961 Parker-Rhodes invented the combinatorial hierarchy [8] and in subsequent work crystallized his concept of indistinguishables [9]. He starts from bit strings of length 2, and maps closure under XOR using square matrices and binary arithmetic. His abstract scheme gives the three levels discussed above that we have been able to relate to the neutrinos, charged leptons, and quarks of the standard model. Mapping the 127 sets of level 3 which close under XOR using $16 \times 16$ matrices yields strings of length 256 and $2^{127}-1$ sets. Parker-Rhodes' sequence cannot be extended because the $256^{2}$ independent mapping matrices available cannot begin to cover this number of sets.

By extending the Dyson argument to gravitation (Ref. [9]), the termination of the mapping can be attributed to the formation of a black hole containing, initially, $2^{127}+136 \approx 1.7 \times 10^{38} \approx \hbar c / G m_{p}^{2}$ gravitating baryons of protonic mass within $\hbar / m_{p} c$. This assemblage is unstable due to Hawking radiation (which our model produces in quantized form), but if the system started from charge $e$, spin $\frac{1}{2} \hbar$ and baryon number 1 , our baryon-number conserving model insures that it will end up as a gravitationally stabilized proton. From the Zurek-Thorne point of view [10], the $2^{127}+136$ baryon-antibaryon pairs which are lost in this process are just the number of bits of information lost in forming this charged, rotating black hole. Similarly, lepton number conservation allows us to view the electron as gravitationally stabilized. This in turn allows us to use the electron mass in the way we did in calculating the pion and muon masses given in the table.

We have now identified five universal constants $\left(\hbar, c, m_{p}, G, e^{2}\right)$. Dimensional analysis allows us to take any three from experiment in order to connect our scheme to the arbitrary units of mass, length, and time in which physicists express the results of measurement. Taking these to be $\hbar, c$ and $m_{p}$ and applying the McGoveran corrections we get the fine structure constant to seven significant figures and $G$ in agreement with experiment, as indicated in the table.

We have now shown why, in our model, the electron and proton are stable. But their mass ratio will still depend on their electromagnetic and weak interactions. Parker-Rhodes originally computed the $m_{p} / m_{e}$ ratio using an interesting mixture of classic and combinatorial ideas (Ref. [11]). McGoveran [11] has supplied an alternative combinatorial argument, which appears to me to attribute most of the mass of the proton to the strong (QCD) interactions.
Then the Parker-Rhodes calculation of $m_{p} / m_{e}$ can be carried through using either the electromagnetic coupling constant $\left(\alpha=e^{2} / \hbar c\right)$ or the Fermi (weak) coupling constant. Self-consistency then requires that the two mass ratios so calculated be equal, to a good approximation. Independent of the details, the "structural" factor, in my interpretation of the formulae is the same in both calculations.

To be more explicit, we refer to our original discussion of the Parker-Rhodes $m_{p} / m_{e}$ calculation (Ref. [3], main text). There we noted that dimensional analysis shows that $m_{p} / m_{e}=137 /\langle x(1-x)\rangle\langle 1 / y\rangle$ where " 137 " $=\hbar c / e^{2}$ and the geometrical factor comes from a statistical calculation of the creation and reabsorption of proton-antiproton pairs in 3-space, due to the coupling of the electron to proton-antiproton pairs via virtual $\gamma$-rays with probability $1 / 137$. If we use the Fermi interaction due to virtual weak vector bosons rather than $\gamma$-rays to create and reabsorb the same proton-antiproton pairs, thus replacing $e^{2} / \hbar c$ by $G_{F} m_{p}^{2} / \hbar c$, appropriately normalized, the rest of the calculation remains the same. In particular, the geometrical (or "structure") factor $\langle x(1-x)\rangle\langle 1 / y\rangle$ is identical. Since both the electromagnetic and the weak calculations are perturbative in the respective coupling constants, the two calculations must specify the same mass for the electron in ratio to the proton mass, up to the neglect of terms of order $\left[e^{2} / \hbar c\right] \times\left[G_{F} m_{p}^{2} / \hbar c\right]$. Equating the two results, the geometrical factor cancels out and we arrive at the same connection between $e^{2}$ and $G_{F}$ which weak-electromagnetic unification at the "tree level" also predicts. Calculated parameters are given in the table. It is to be emphasized that our understanding of weak-electromagnetic unification does not require -indeed give no indication of the existence of - the unobserved "Higgs boson."

\section{Content strings, discrete space-time, and cosmology}

One distinction (an advantage from my point of view) of using program universe rather than the Parker-Rhodes mapping to generate our strings is that it continues to generate growing content strings after the labels of the known particles are gravitationally stabilized and fixed. We now sketch how these can be used to represent particle scattering processes to requisite accuracy.

We have shown above that $|\mathbf{a} \oplus \mathbf{b}|=a+b-$ $2\langle a \mid b\rangle$. It follows that $|\mathbf{a} \oplus \mathbf{b}| \leq a+b$. Further, the smallest value that $|\mathbf{a} \oplus \mathbf{b}|$ can take on occurs when the maximum number of cancellations between the non-null elements in the two strings take place, i.e., when the maximum number of instances of $a_{w}=1$, $b_{w}=1$ and hence $a_{w}-b_{w}=0$ occur. Clearly, in 
this case $|\mathbf{a} \oplus \mathbf{b}|=|a-b|$ and we have proved that $|a-b| \leq|a \oplus \mathbf{b}| \leq a+b$. It follows immediately that any three strings which discriminate to the null string- $\mathbf{a} \oplus \mathbf{b} \oplus \mathbf{c}=\mathbf{0}$ - specify a triangle with integer sides given by the thre Hamming measures $a, b, c$. This follows from the fact that these three integers satisfy the triangle inequalities $|a-b| \leq c \leq a+b$, cyclic on $a, b, c$, as we have just proved above. Similarly, the constraint $\mathbf{a} \oplus \mathbf{b} \oplus \mathbf{c} \oplus \mathbf{d}=\mathbf{0}$ and the restriction $a+b+c<d$ specifies (up to a choice of chirality) a tetrahedron with arbitrary integer edges $a, b, c$. We have already shown that $a, b, b, c$ and $c, a$ specify triangles with the third side of length $|\mathbf{a} \oplus \mathbf{b}|,|\mathbf{b} \oplus \mathbf{c}|$ and $|c \oplus a|$ respectively. The fourth side is obviously also a triangle with sides of length $|\mathbf{a} \oplus \mathbf{b}|,|\mathbf{b} \oplus \mathbf{c}|$ and $|\mathbf{c} \oplus \mathbf{a}|$. If we interpret the corners of the tetrahedron as counter firings, and assume that (for example) the time it takes a light signal to go along side $a$ to the $a b c$ vertex of the tetrahedron and back along side $b$ is the same as the time it takes a particle to go along the third side of length $|\mathbf{a} \oplus \mathbf{b}|$; this speed is $v_{a b}=\frac{|\mathbf{a} \oplus \mathbf{b}|}{a+b} c<$ c. Clearly this construction has a Lorentz invariant kinematic significance for finite and discrete rotations and boosts which preserve the integer character of the description [12]. The non-commutativity of finite rotations then implies the non-commutativity of position-velocity measurements, which in turn implies relativistic commutation relations. These commutation relations in turn allow us to model a finite and discrete version of relativistic quantum mechanics when the minimum length interval between events is $h / m c$.

To give this construction a dynamic significance, we recall that the energy-momentum structure for four particle processes with four-momenta $p_{i}=\left(E_{i}, \vec{p}_{i}\right)$, $i \in 1,2,3,4(c=1)$ can be specified by the three scalar Mandelstam invariants $s, t, u$ [13]. These are defined for the process $1+2 \rightarrow 3+4$ by $s=\left(p_{1}+\right.$ $\left.p_{2}\right)^{2}=\left(p_{3}+p_{4}\right)^{2}=m_{1}^{2}+2 E_{1} E_{2}-2 \vec{p}_{1} \cdot \vec{p}_{2}+m_{2}^{2}$; $t=\left(p_{1}-p_{3}\right)^{2}=\left(p_{2}-p_{4}\right)^{2}=m_{1}^{2}-2 E_{1} E_{3}+2 \vec{p}_{1} \cdot \vec{p}_{3}+m_{3}^{2}$; $u=\left(p_{1}-p_{4}\right)^{2}=\left(p_{2}-p_{3}\right)^{2}=m_{1}^{2}-2 E_{1} E_{3}+2 \vec{p}_{1}$. $\vec{p}_{4}+m_{4}^{2}$. Note that $s, t, u$ are not independent because (on shell) energy-momentum conservation guarantees that $s+t+u=m_{1}^{2}+m_{2}^{2}+m_{3}^{2}+m_{4}^{2}$. Assume that energies and momenta are integers when measured in units of some smallest mass $\Delta m$, and that the individual particle invariants on mass shell are given by $s_{i}=E_{i}\left(E_{i}+1\right)-\vec{p}_{i} \cdot \vec{p}_{i}=m_{i}^{2}$. Here finite and discrete Lorentz boost invariance is preserved for any integer value of $p$ in the range $-E \leq p \leq+E$ in the same way that the finite and discrete angular momentum components in units of $\hbar$ preserve the rotational invariance of $j(j+1)-j_{z}^{2}=(1 / 2)\left(j_{+} j_{-}+j_{-} j_{+}\right)=$ $j_{x}^{2}+j_{y}^{2}$. Representation in terms of bit-strings and construction of a four-particle relativistic scattering theory of the Faddeev-Yakubovsky type, using sums rather than integrals, is tedious but straightforward. The Mandelstam invariants emerge in a natural way, as does crossing symmetry. Details will be presented elsewhere.

We have seen that program universe can generate labels which can in turn be used it to model the quantum numbers eventually associated with "electric charge" or "visible matter". This tentative identification closes when we have reached the 127 labels of level 3 and the 256 labels which fix a particular representation of the standard ( 3 generation) model of quarks and leptons. During this process we will encounter the 10 labels of levels 1 and 2, statistically, 12.7 times more frequently than the labels associated with electric charge. Once label length exceeds 256 and we start constructing our model of space-time, with the gravitational constant also fixed, this class of 10 uncharged labels can form gravitationally bound "dark matter" composed of neutrinos, photons and gravitons. The 10 labels can now specify two spin states for the neutrinos, two for the spin 1 photons, five for the spin two gravitons, and a tenth for our quantized version of Newtonian gravitation. Thanks to lepton number conservation, this uncharged (dark) "matter" can coalesce around finite numbers of neutrinos or (but not and!) anti-neutrinos. We have yet to compute the fluctuations in program universe which will (statistically) predict the distribution of masses in this dark matter.

The random choice of the concatenated bits in program universe guarantees that, to a first approximation, all strings will contain an equal number of zeros and ones, and hence that the universe will have about the same number of fermions and antifermions. We expect these to annihilate to (eventually) photons, but fermionic labels cannot persist unless there is departure from equality of zeros and ones by at least one unit for the total collection of labels. Since we have $(256)^{4}$ possible types of baryon-number conserving collisions which lead to photons, we expect the baryon to photon ratio to be $256^{-4}$, which is about right. After fireball time (transition from an optically thick to an optically thin universe) we expect a conventional, matter dominated expansion. Using the number of distinct labels $\left(2^{127}+136\right)$, the number of baryons expected from the pairwise collisions is the square of that number. If this is all in what is observationally believed to be baryonic matter, we expect this to be about $2 \%$ of the critical mass, which is about what is observed. Whether we can meet the power spectrum fluctuations in the background radiation observed by COBE and the light element isotope mass ratios ("deuteronomy") remains to be seen. Preliminary values of the parameters are collected in the cosmological section of the table. 


\section{Electromagnetism and gravitation}

The Feynman-Dyson [14] -Tanimura [15] proof of the Maxwell and Einstein equations for free space electromagnetic and gravitational fields makes sense when we note that field strength per unit mass or charge is defined by the acceleration of a single test particle. [16] This allows us to use a scale invariant space-time theory based on measuring finite and discrete intervals between events (such as counter firings) and fixed measurement accuracy $\Delta x, \Delta t$ down to intervals which involve (directly or indirectly) the production of particle-gparticle pairs. This lower bound on classical, relativistic scale invariance arises because of the complete breakdown of the concept of "test particle" and hence of "classical, relativistic field" once the phenomenon of pair creation prevents us from giving meaning to what we mean by a single particle. The Dyson paper is admittedly paradoxical, and Tanimura's paper is at least as ambiguous. However, by using "scale invariance bounded from below" and a significant extension of the calculus of finite differences to non-commuting coordinate shifts, L.H.Kauffman and HPN (in preparation), believe we have succeeded in providing a rigorous underpinning for these derivations, showing that our theory contains electromagnetism and gravitation as macroscopic approximations.

\section{Caveat and conclusion}

We warn the reader that detailed and rigorous mathematical proof of some of the statements made above is still missing. We wish to thank David McGoveran for pointing out to us that this caveat is particularly relevant for the use we make of the corrections he derived in the context of the combinatorial hierarchy construction. For him, constructing our bit-strings using program universe and bringing in the identification of the labels form "outside" - i.e., from known facts about quantum number conservation in particle physics - amounts to creating a different theory. While we have confidence that mixing up the two approaches in the way we have can, eventually, be justified in a compelling way, it may well turn out that our confidence in this outcome is overly optimistic.

To summarize, by using a simple algorithm and detailed physical interpretation, we believe we have constructed a self-organizing universe which bears a close resemblance to the one in which physicists think we live. It is not "self-generating"-unless one grants that the two postulates with which Parker-Rhodes begins his book on the "inevitable universe", namely: "Something exists!" and "This statement conveys no information" suffice to explain why our universe started up.

\section{References}

[1] H.P. Noyes, "OPERATIONALISM REVISITED: Measurement Accuracy, Scale Invariance and the Combinatorial Hierarchy," Physics Philosophy Interface (in press).

[2] H.P. Noyes, "STAPP's QUANTUM DUALISM: The James/Heisenberg Model of Consciousness," in Mind-Body Problem and the Quantum; Proc. ANPA WEST 10, F. Young, ed. 112 Blackburn Ave., Menlo Park, CA 94025.

[3] J.Amson, Appendix in T.Bastin, H.P.Noyes, C.W. Kilmister, and J.Amson, Int.J.Theor.Phys., 18, 455 (1979).

[4] M.J. Manthey, in H.P. Noyes, "ON THE CONSTRUCTION OF RELATIVISTIC QUANTUM MECHANICS: A Progress Report", SLAC-PUB4008, June 1986, pp. 101-110.

[5] H.P. Noyes and D.O. McGoveran, Physics Essays, 2, 76 (1989).

[6] D.O. McGoveran and H.P. Noyes, Physics Essays 4, 115 (1991).

[7] D.O. McGoveran and H.P. Noyes, in Discrete and Combinatorial Physics (Proc. ANPA 9), H.P. Noyes, ed., ANPA WEST, 409 Leland Ave, Palo Alto, CA 94306; see also Ref. 5, p. 91.

[8] F.J. Dyson, Phys. Rev. 85, 631 (1952).

[9] H.P. Noyes, "Non-Locality in Particle Physics," SLAC-PUB-1405 (1975).

[10] T. Bastin, "On the Scale Constants of Physics," Studia Philosophica Gandensia, 4, 77 (1966).

[11] A.F. Parker-Rhodes, "The Theory of Indistinguishables," Synthese, 150, Reidel, Dordrecht, Holland (1981); - "The Inevitable Universe" (unpublished).

[12] W.H. Zurek and K.S. Thorne, Phys. Rev. Lett. 54, 2171 (1985).

[13] D.O. McGoveran, in Alternatives in Physics and Biology, M.J. Manthey, ed., ANPA 12, F. Abdullah, City Univ., London EC1V 0HB, pp. 15-17.

[14] H.P. Noyes, "The RQM Triangle", in Reconstructing the Object; Proc. ANPA WEST 8, F. Young, ed., ANPA WEST, 112 Blackburn Ave., Menlo Park, CA 94025.

[15] "Review of Particle Properties," Phys. Rev. D 45, 1 June 1992, Part II, p. III.50.

[16] F.J. Dyson, Am. J. Phys, 58, 209 (1990).

17] S. Tanimura. Annals of Physics, 220, 229 (1992).

[18] "On the Measurability of Electromagnetic Fields", in Marshal Memorial Volume, E.G. Sudarshan, ed., World Scientific (in press). 